\title{
Chenodeoxycholate induction of mitochondrial permeability transition pore is associated with increased membrane fluidity and cytochrome $c$ release: protective role of carvedilol
}

\author{
Anabela P. Rolo, Paulo J. Oliveira, Antonio J. Moreno, Carlos M. Palmeira* \\ Department of Zoology, Center for Neurosciences and Cell Biology of Coimbra, University of Coimbra, 3004-517 Coimbra, Portugal
}

Received 5 August 2002; received in revised form 2 January 2003; accepted 3 January 2003

\begin{abstract}
Chenodeoxycholate (CDCA) is a primary bile acid mostly implicated in cholestatic liver injury. In this study, we have investigated the involvement of membrane fluidity and cytochrome $c$ release in CDCA-induced mitochondrial permeability transition (MPT), and the preventive role of carvedilol. Treatment of calcium-loaded hepatic mitochondria with CDCA was found to cause osmotic swelling and release of cytochrome $c$, associated with an increase in membrane fluidity, in both protein and lipid regions. Carvedilol and cyclosporine $\mathrm{A}(\mathrm{CyA})$ reduced both cytochrome $c$ release and alterations in membrane fluidity induced by CDCA. The hydroxylated metabolite of carvedilol, BM-910228, had no effect. Thus, modulation of membrane fluidity, plays an important role in MPT pore opening promoted by CDCA. As a result, we have delineated a pathway for the preventive role of carvedilol in mitochondrial dysfunction induced by CDCA.
\end{abstract}

(C) 2003 Elsevier Science B.V. and Mitochondria Research Society. All rights reserved.

Keywords: Chenodeoxycholate; Carvedilol; Mitochondria; Cholestasis; Cardiomyopathy; Mitochondrial permeability transition; Membrane fluidity

\section{Introduction}

Bile formation is a vital function of the liver and its impairment is central in the pathogenesis of cholestasis. In cholestatic disease, intrahepatic accumulation of toxic bile acids promote hepatic injury (Greim et al., 1972). Additionally, a possible relationship between plasma accumulation of bile acids and the cardiomyopathy of cholestatic liver disease has been proposed (Joubert, 1978). CDCA is a primary bile acid mostly

\footnotetext{
* Corresponding author. Tel.: +351-239-834729; fax: +351239-826798.

E-mail address: palmeira@ci.uc.pt (C.M. Palmeira).
}

implicated in liver injury. Hepatic levels of CDCA increase 20-fold during cholestasis and are greater than those of other toxic bile acids such as lithocholate and deoxycholate (Greim et al., 1973).

Although the cellular mechanisms involved in bile acid-induced injury are still under investigation, it is known that hydrophobic bile acids are particularly hepatotoxic. Due to their membrane-active, detergentlike properties, the cytotoxicity of bile acids has been correlated to the degree of lipophilicity of the different molecular species (Attili et al., 1986). Mechanisms implicated in the toxicity of bile acids include stimulation of lipid peroxidation (Sokol et al., 1993) 
and induction of mitochondrial dysfunction (Schaffner et al., 1971; Krahenbuhl et al., 1992; Gores et al., 1998; Rolo et al., 2000).

Carvedilol, a multiple-action neuro-hormonal antagonist with known cardioprotective effects, has been previously described (Rolo et al., 2001) as a protective agent against liver mitochondrial damage induced by CDCA.

Induction of the mitochondrial permeability transition (MPT) is widely implicated in the pathophysiology of cell death caused by a number of agents (Lemasters et al., 1998). It is characterized by an increase in non-specific permeability of the inner membrane to low molecular weight solutes leading to mitochondrial membrane depolarization, mitochondrial calcium release, mitochondrial swelling and inhibition of oxidative phosphorylation (Zoratti and Szabo, 1995). Swelling then leads to outer membrane rupture and release of cytochrome $c$ and other proapoptotic factors (Lemasters, 1999). The MPT is suggested to reflect the opening of a pore composed of assorted elements, the formation of which is inhibited by cyclosporine A (CyA) (Broekemeier et al., 1989). An association between MPT and changes in mitochondrial membrane fluidity has been described (Ricchelli et al., 1999), probably related with conformational changes during the assembly of membrane-spanning proteins into a non-specific pore.

In this study, we examined CDCA-induced MPT events such as mitochondrial swelling, cytochrome $c$ release, and changes in mitochondrial membrane fluidity, as probed by membrane labeling with fluorescent dyes. We therein investigated the effects of carvedilol and its metabolite BM-910228 on the CDCA-induced MPT in rat liver mitochondria.

\section{Materials and methods}

\subsection{Chemicals}

CDCA, used as ethanolic solution, hematoporphyrin IX (HP), and 1,6-diphenyl-1,3,5-hexatriene (DPH), were purchased from Sigma Chemical Co. Carvedilol and BM-910228 were obtained from Boehringer. All other chemicals were of the highest grade of purity commercially available.

\subsection{Isolation of mitochondria}

Mitochondria were isolated from liver of male Wistar rats by conventional methods (Gazotti et al., 1979) with slight modifications. Homogenization medium contained $250 \mathrm{mM}$ sucrose, $10 \mathrm{mM}$ HEPES (pH 7.4), $0.5 \mathrm{mM}$ EGTA, and $0.1 \%$ fat-free BSA. EGTA and BSA were omitted from the final washing medium, adjusted at $\mathrm{pH}$ 7.4. The mitochondrial pellet was washed twice, suspended in the washing medium, and immediately used. Protein content was determined by the biuret method (Gornall et al., 1949) calibrated with BSA.

\subsection{Measurement of mitochondrial permeability transition}

Mitochondrial swelling was estimated by changes in light scattering as monitored spectrophotometrically at $540 \mathrm{~nm}$ (Palmeira and Wallace, 1997). The reaction medium was stirred continuously and the temperature maintained at $25^{\circ} \mathrm{C}$. The experiments were started by the addition of $1 \mathrm{mg}$ of mitochondria to a final volume of $2 \mathrm{ml}$ of the standard incubation medium, supplemented with $2 \mu \mathrm{M}$ rotenone, $0.5 \mu \mathrm{g}$ of oligomycin, and $5 \mathrm{mM}$ succinate. Calcium $(35 \mu \mathrm{M}$ $\mathrm{CaCl}_{2}$ ) was added prior to all the other compounds.

\subsection{Cytochrome c assay}

The release of cytochrome $c$ by isolated mitochondria was determined spectrophotometrically at $410 \mathrm{~nm}$. The reactions were carried out at $25^{\circ} \mathrm{C}$, in $2 \mathrm{ml}$ of the standard incubation medium, supplemented with $2 \mu \mathrm{M}$ rotenone, $0.5 \mu \mathrm{g}$ of oligomycin, and $5 \mathrm{mM}$ succinate. Mitochondria $(2 \mathrm{mg} / \mathrm{ml})$ were suspended with gentle shaking for $30 \mathrm{~min}$, in the presence of different additions as shown in the legends to the figures. After incubation, cytochrome $c$ release was evaluated as previously described (Appaix et al., 2000). Quantification was achieved by employing a standard curve. Cytochrome $c$ release induced by the test agents was expressed as a percent of total release, which was evaluated after incubation of mitochondria in a hypotonic medium. 


\subsection{Mitochondrial membrane fluidity analysis}

Membrane fluidity changes induced by the test agents were evaluated by assessing the fluorescence polarization of mitochondria bound dyes. The $P$ value for the probes fluorescence was used as a measure of membrane fluidity, with increased polarization indicating a decreased extent of phospholipid acyl chain motion within the membrane (Kawato et al., 1977). Two probes were used: HP (excitation $520 \mathrm{~nm}$; emission $626 \mathrm{~nm}$ ) and DPH (excitation $340 \mathrm{~nm}$; emission $460 \mathrm{~nm}$ ), that monitor the fluidity of different regions of membranes. With the DPH probe, fluidity of highly hydrophobic lipid phases is evaluated (Kawato et al., 1977). HP has been described as accumulating in very-polar, specific, localized lipid regions, such as protein regions in the inner mitochondrial membrane (Ricchelli et al., 1995). HP (final concentration of $3 \mu \mathrm{M}$ ) was injected into stirred mitochondrial suspensions $(0.5 \mathrm{mg} / \mathrm{ml}$ of the standard incubation medium) and the mixture incubated for 2 min before measurements; DPH (final concentration of $2 \mu \mathrm{M}$ ) required longer incubation times $(1 \mathrm{~h})$. The different additions are shown in the legends to the figures.

\subsection{Statistical analysis}

All data are expressed as the mean \pm SEM for four independent experiments. Statistical evaluation was performed using the two-tailed paired Student's $t$ test. A $P$ value $<0.05$ was considered statistically significant.

\section{Results}

\subsection{CDCA-induced mitochondrial swelling}

Fig. 1 reports mitochondrial swelling due to MPT induction by CDCA $(50 \mu \mathrm{M})$, in succcinate-energized, rat liver mitochondria. In the presence of calcium $(35 \mu \mathrm{M})$, hepatic mitochondria underwent a large amplitude decrease of light scattering, upon addition of CDCA. No swelling was observed in the control experiment (Fig. 1) following calcium addition, showing that mitochondria maintained their permeability barrier. CyA prevented CDCA-

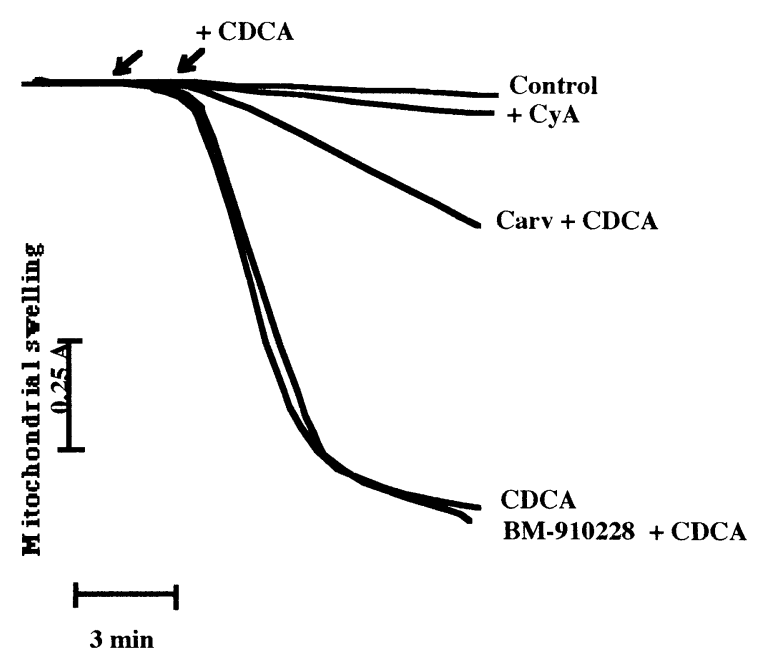

Fig. 1. Effects on mitochondrial swelling. The incubation medium (final volume $2 \mathrm{ml}$ at $25^{\circ} \mathrm{C}$ ) contained $200 \mathrm{mM}$ sucrose, $10 \mathrm{mM}$

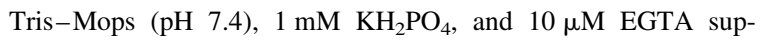
plemented with $3 \mu \mathrm{M}$ rotenone, $0.5 \mu \mathrm{g}$ oligomycin, and $5 \mathrm{mM}$ succinate. The experiments were started by the addition of $1 \mathrm{mg}$ of mitochondria (not shown). After establishing a baseline, calcium was added $(35 \mu \mathrm{M})$. Where indicated (first arrow), CyA $(1 \mu \mathrm{M})$, carvedilol (Carv, $10 \mu \mathrm{M})$, or BM-910228 $(10 \mu \mathrm{M})$ were included in the reaction medium prior to $50 \mu \mathrm{M}$ CDCA. The traces are typical of several independent experiments with four different mitochondrial preparations.

induced mitochondrial swelling, indicating that the decrease in absorbance was the result of the calciumdependent MPT. A similar result was observed after pre-incubation with carvedilol $(10 \mu \mathrm{M})$, whereas BM$910228(10 \mu \mathrm{M})$ had no significant effect (Fig. 1).

\subsection{CDCA-induced cytochrome c release}

Fig. 2 shows the release of cytochrome $c$ into the medium after MPT induction by CDCA, in calcium loaded mitochondria. A remarkable increase in cytochrome $c$ in the medium was observed in the presence of both calcium and CDCA. Similar to the swelling experiments, CyA had a preventive role, associated with inhibition of the MPT. Carvedilol was also effective, since pre-incubation with this compound strongly prevented CDCA-induced MPT and the associated cytochrome $c$ release. In contrast, BM910228 had no effect on the mitochondrial response to CDCA addition. 


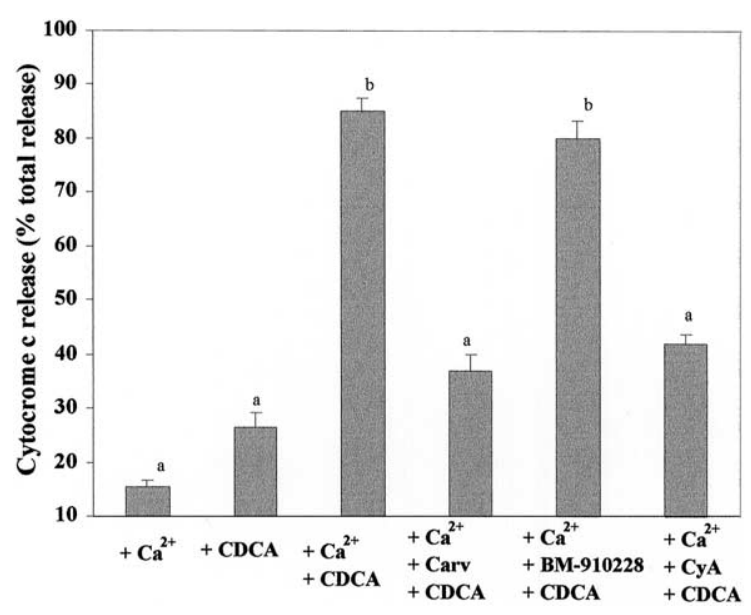

Fig. 2. Effects on cytochrome $c$ release. Mitochondria were incubated in $2 \mathrm{ml}$ of standard incubation medium, supplemented with $3 \mu \mathrm{M}$ rotenone, $0.5 \mu \mathrm{g}$ oligomycin, and $5 \mathrm{mM}$ succinate. As shown in the figure, different test agents were added: calcium $(35 \mu \mathrm{M})$ and/or CDCA $(50 \mu \mathrm{M})$; where indicated CyA $(1 \mu \mathrm{M})$, carvedilol (Carv, $10 \mu \mathrm{M})$, or BM-910228 $(10 \mu \mathrm{M})$ was included in the reaction medium prior to CDCA. After incubation (20 min), cytochrome $c$ release into the medium was evaluated as previously described (Appaix et al., 2000). The effects of the test agents are expressed as percent of total release, taking into account the complete release of cytochrome $c$ following incubation of mitochondria in a hypotonic medium. Data represent the mean \pm SEM of measurements from four different mitochondrial preparations. Values that share the same letter are not statistically different $(P<0.05)$, compared by the two-tailed paired Student's $t$ test.

\subsection{CDCA-induced alterations in membrane fluidity}

To determine whether CDCA-induced mitochondrial swelling was related to changes in membrane fluidity, DPH and HP fluorescence polarization values were analyzed spectrofluorometrically (Fig. 3A, B , respectively). DPH reflects the fluidity of all membrane layers (Kawato et al., 1977); HP typically probes protein regions (Ricchelli et al., 1995). In the presence of CDCA, there was a decrease in polarization values for DPH (Fig. 3A), showing that CDCA by itself causes an increase in membrane fluidity. Furthermore, in the presence of both calcium and CDCA, there was a significant increase in membrane fluidity, which appeared to parallel the light scattering change. The strict correlation between changes in membrane fluidity and the increase of permeability due to MPT induction, was demonstrated by the inhibitory effect of CyA. Carvedilol had a similar
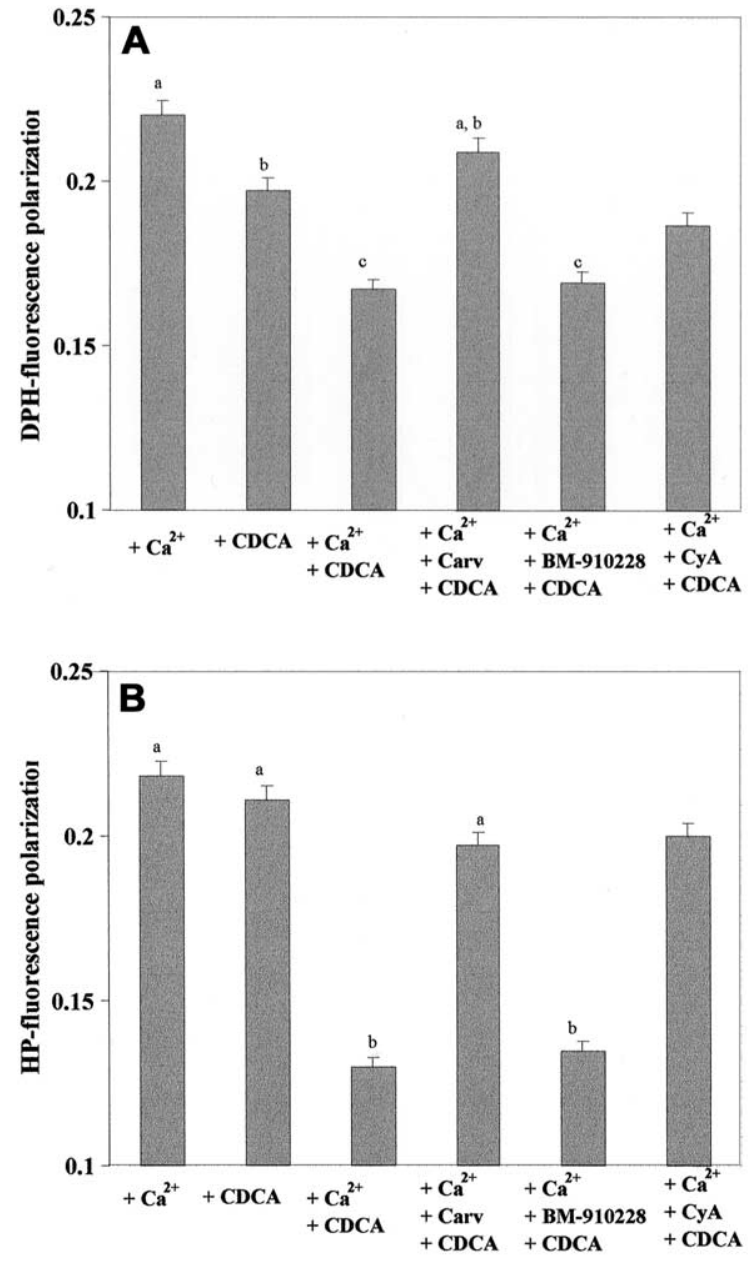

Fig. 3. (A,B) Effects of CDCA on mitochondrial membrane fluidity as probed by DPH (A) and HP (B). Following incubation with the probes, the different test agents were added to mitochondria suspended in $2 \mathrm{ml}$ of standard incubation medium (supplemented with $3 \mu \mathrm{M}$ rotenone, $0.5 \mu \mathrm{g}$ oligomycin, and $5 \mathrm{mM}$ succinate). The conditions were the same as described for Fig. 2. The changes in polarization were monitored for a period of time similar to the one considered in the swelling experiment $(20 \mathrm{~min})$. The effects of the test agents are expressed as changes in the polarization values. Data represent the mean \pm SEM of measurements from four different mitochondrial preparations. Values that share the same letter are not statistically different $(P<0.05)$, compared by the two-tailed paired Student's $t$ test.

effect. In contrast, in the presence of BM-910228, the changes in DPH polarization were similar to those observed in the presence of both calcium and CDCA.

Fig. 3B reports the effect of CDCA-induced MPT on the membrane fluidity, as probed by HP. It has 
been described that HP accumulates in specific, localized lipid regions, perhaps in the boundary lipids area surrounding some inner-mitochondrial carriers (Ricchelli et al., 1995). After CDCA addition to calcium-loaded mitochondria, there was a decrease in polarization values, which was prevented by both CyA and carvedilol. BM-910228 had no effect.

\section{Discussion}

Our previous study (Rolo et al., 2001) has demonstrated that carvedilol displays a novel role, preventing mitochondrial dysfunction associated with the MPT induction by CDCA. In the present study, we further demonstrate that MPT induction by CDCA is associated with changes in mitochondrial membrane fluidity, that are inhibited by carvedilol but not by its hydroxylated analog, BM-910228. So, prevention by carvedilol could be related with the physical stabilization of mitochondrial membrane, as opposed to disturbance induced by CDCA. Interestingly, work made by others (Rodrigues et al., 1999, 2000) has demonstrated that hydrophilic bile acids, such as ursodeoxycholic (UDCA) and its taurine derivative (TUDC), are inhibitors of apoptosis via stabilization of the mitochondrial membrane, through pathways that are independent of the MPT. In fact, these studies regarding induction of apoptosis through a mitochondrial-dependent pathway, have showed that, although there was no protection by CyA, UDCA or TUDC, were able to prevent cell death. The proposed mechanism for hydrophilic bile acids protection correlates inhibition of mitochondrial depolarization and outer membrane disruption, together with modulation of Bax translocation from cytosol to mitochondria. This similarity of cytoprotective actions of both carvedilol and hydrophilic bile acids may reflect modulation of membrane perturbation due to their lipophilicity. In contrast, and in concordance with our results, CyA blocked MPT and also prevented mitochondrial swelling and reduced the efflux of cytochrome $c$ induced by deoxycholic acid, a hydrophobic bile acid, in primary rat hepatocytes (Rodrigues et al., 1999). This suggests that, in contrast to hydrophilic bile acids, cytotoxicity of hydrophobic bile acids involve mitochondrial disruption and cytochrome $c$ release primarily through MPT opening.

Hepatocellular damage in cholestasis is characterized by intracellular accumulation of bile acids (Greim et al., 1972). Furthermore, cardiomyopathy has been associated with cholestatic liver disease, probably due to increased plasma levels of bile acids (Joubert, 1978). A number of observations suggest that mitochondria are a primary target of chemical induced injury and that their dysfunction ultimately leads to cell death (Petit et al., 1995; Wallace and Starkov, 2000). Both morphological and biochemical observations support the concept that bile acids may be cytotoxic by causing mitochondrial dysfunction (Schaffner et al., 1971; Krahenbuhl et al., 1992; Sokol et al., 1993; Gores et al., 1998; Rolo et al., 2000), particularly at the level of MPT induction. Besides impairment of mitochondrial bioenergetics, permeabilization of the mitochondrial membrane releases several proteins, including cytochrome $c$ (Kluck et al., 1997). In mitochondria, cytochrome $c$ plays an essential role in generation of mitochondrial transmembrane potential. Additionally, the release of cytochrome $c$ from the inter-membrane space into cytosol also leads to the initiation of a prompt apoptotic cascade (Li et al., 1997).

Here, we report that carvedilol and CyA prevent CDCA-induced MPT and the associated cytochrome $c$ release. Moreover, using the hydroxylated metabolite of carvedilol, shown to have higher antioxidant activity than carvedilol (Yue et al., 1994), we did not detect protection against CDCA-induced membrane disturbance. Thus, these results are consistent with a protection that occurs via the inhibition of the MPT, and that is not related with the antioxidant properties. However, the results do not exclude a direct interaction with the MPT assembly, being carvedilol able to inhibit mitochondrial membrane disturbance caused by CDCA to calcium-loaded mitochondria. This difference may reflect the different properties of both carvedilol and BM-910228 in terms of membrane insertion. Due to its great lipophilicity, carvedilol has a considerable tendency for membrane insertion (Cheng et al., 1996) and may disturb the correct assembly of the pore proteic components. This would explain the similar results obtained with CyA and carvedilol. Indeed, the membrane fluidity studies, reflecting the dynamic properties of mitochondrial 
membranes, regarding protein regions, as probed by HP, show the strict correlation between the inhibitory effect of carvedilol and the changes in the polarization values due to pore opening.

We have observed that CDCA renders mitochondrial membranes more permeable, as shown by the increased membrane fluidity, probed by DPH. It is well established that hydrophobicity, as demonstrated by its close correlation with the partition coefficient of compounds, is often associated with biological action (Hansch and Dunn, 1972). Bile acids, in particular the lipophilic dihydroxy, such as CDCA, and monohydroxy species, are surface-active substances and can damage biological membranes (Scholmerich et al., 1984). Some reports, regarding in vitro studies, have been shown direct effects of bile acids on membrane fluidity (Isaacson and van Thiel, 1988; Schroder et al., 1996), associated with increased proton permeability of the membranes (Zhao and Hirst, 1990; Schroder et al., 1996). This in accordance with our results, since besides induced increased fluidity due to MPT induction, CDCA also decreased DPH polarization in non-calcium-loaded mitochondria. Furthermore, CyA did not prevent this effect. However, CyA completely protected against the calcium-dependent swelling, cytochrome $c$ release and HP-probed fluidity changes. So, the already described stimulation of state four respiration in isolated mitochondria (Rolo et al., 2000), caused by CDCA, may be a consequence of increased membrane fluidity.

In conclusion, our results indicate that alterations in membrane fluidity are events involved in the protection afforded by carvedilol against CDCAinduced MPT, characterized by osmotic swelling and cytochrome $c$ release. The disturbance of mitochondrial membrane by CDCA could lead to impairment of mitochondrial function, and subsequent cell dysfunction observed during cholestasis. Since bile acid accumulation has been proposed as a causative factor for hepatic cardiomyopathy, carvedilol administration may potentially prevent mitochondrial dysfunction associated with cardiomyopathy in cholestatic liver disease.

\section{Acknowledgements}

This work was supported by FCT (Fundação para a
Ciência e Tecnologia, Lisbon, Portugal); Research Project POCTI/CBO/42486/2001. A.P.R and P.J.O are recipients of PRAXIS grants, XXI/BD/21454/99 and XXI/21494/99, respectively, from the Fundação para a Ciência e Tecnologia (FCT), Lisbon, Portugal.

\section{References}

Appaix, F., Minatchy, M.-N., Riva-Lavieille, C., Olivares, J., Antonsson, B., Saks, V.A., 2000. Rapid spectrophotometric method for quantitation of cytochrome $c$ release from isolated mitochondria orpermeabilized cells revisited. Biochim. Biophys. Acta 1457, 175-181.

Attili, A.F., Angelico, M., Cantafora, A., Alvaro, D., Capocaccia, L., 1986. Bile acid-induced liver toxicity: relation to the hydrophobic-hydrophilic balance of bile acids. Med. Hypotheses 19, 57-69.

Broekemeier, K.M., Dempsey, M.E., Pfeiffer, D.R., 1989. Cyclosporin $\mathrm{A}$ is a potent inhibitor of the inner membrane permeability transition in liver mitochondria. J. Biol. Chem. 264, 7826-7830.

Cheng, H.-Y., Randall, C.S., Holl, W.W., Constantinides, P.P., Yue, T.-L., Feuerstein, G.Z., 1996. Carvedilol-liposome interaction: evidence for strong association with the hydrophobic region of the lipid bilayers. Biochim. Biophys. Acta 1284, 20-28.

Gazotti, P., Malmstron, K., Crompton, M., 1979. Membrane biochemistry. In: Carafoli, E., Semenza, G. (Eds.), A Laboratory Manual on Transport and Bioenergetics, Springer, New York, NY.

Gores, G.J., Miyoshi, H., Botla, R., Aguilar, H.I., Bronk, S.F., 1998. Induction of the mitochondrial permeability transition as a mechanism of liver injury during cholestasis: a potential role for mitochondrial proteases. Biochim. Biophys. Acta 1366, 167-175.

Gornall, A.G., Bardawill, C.J., David, M.M., 1949. Determination of serum proteins by means of the biuret reaction. J. Biol. Chem. 177, 751-766.

Greim, H., Trulzsch, D., Czygan, P., et al., 1972. Mechanism of cholestasis. 6 . Bile acids in human livers with or without biliary obstruction. Gastroenterology 63, 846-850.

Greim, H., Czygan, P., Schaffner, F., Popper, H., 1973. Determination of bile acids in needle biopsies of human liver. Biochem. Med. 8, 280-286.

Hansch, C., Dunn, W.J. III, 1972. Linear relationships between lipophilic character and biological activity of drugs. J. Pharmacol. Sci. 61, 1-19.

Isaacson, Y., Van Thiel, D.H., 1988. Increased membrane fluidity and cholestasis: associated but not linked consequences of estrogen treatment. J. Lab. Clin. Med. 112, 663-666.

Joubert, P., 1978. Cholic acid and the heart: in vitro studies of the effect on heart rate and myocardial contractility in the rat. Clin. Exp. Pharmacol. Physiol. 5, 9-16.

Kawato, S., Kinoshita, K., Ikegami, A., 1977. Dynamic structure of 
lipid bilayers studied by nanosecond fluorescence techniques. Biochemistry 16, 2319-2324.

Kluck, R.M., Bossy-Wetzel, E., Green, D.R., Newmeyer, D.D., 1997. The release of cytochrome $c$ from mitochondria: a primary site for Bcl-2 regulation of apoptosis. Science 275, $1132-1136$.

Krahenbuhl, S., Stucki, J., Reichen, J., 1992. Reduced activity of the electron transport chain in liver mitochondria isolated from rats with secondary biliary cirrhosis. Hepatology 15, 1160-1166.

Lemasters, J.J., Nieminen, A.L., Qian, T., et al., 1998. The mitochondrial permeability transition in cell death: a common mechanism in necrosis, apoptosis and autophagy. Biochim. Biophys. Acta 1366, 177-196.

Lemasters, J.J., 1999. V. Necrapoptosis and the mitochondrial permeability transition: shared pathways to necrosis and apoptosis. Am. J. Physiol. 276, G1-G6.

Li, P., Nijhawan, D., Budihardjo, I., et al., 1997. Cytochrome $c$ and dATP-dependent formation of Apaf-1/caspase-9 complex initiates an apoptotic protease cascade. Cell 91, 479-489.

Palmeira, C.M., Wallace, K.B., 1997. Benzoquinone inhibits the voltage-dependent induction of the mitochondrial permeability transition caused by redox-cycling naphthoquinones. Toxicol. Appl. Pharmacol. 143, 338-347.

Petit, P.X., Lecoeur, H., Zorn, E., Dauguet, C., Mignotte, B., Gougeon, M.L., 1995. Alterations in mitochondrial structure and function are early events of dexamethasone-induced thymocyte apoptosis. J. Cell Biol. 130, 157-167.

Ricchelli, F., Gobbo, S., Jori, S., Salet, C., Moreno, G., 1995. Temperature-induced changes in fluorescence properties as a probe of porphyrin microenvironment in lipid membranes. 2 . The partition of hematoporphyrin and protoporphyrin in mitochondria. Eur. J. Biochem. 233, 165-170.

Ricchelli, F., Gobbo, S., Moreno, G., Salet, C., 1999. Changes of the fluidity of mitochondrial membranes induced by the permeability transition. Biochemistry 38, 9295-9300.

Rodrigues, C.M., Ma, X., Stieers, C.L., Fan, G., Kren, B.T., Steer, C.J., 1999. Ursodeoxycholic acid prevents cytochrome $c$ release in apoptosis by inhibiting mitochondrial membrane depolarization and channel formation. Cell Death Differ. 6, 842-854.

Rodrigues, C.M., Stieers, C.L., Keene, C.D., et al., 2000. Tauroursodeoxycholic acid partially prevents apoptosis induced by 3-nitropropionic acid: evidence for a mitochondrial pathway independent of the permeability transition. J. Neurochem. 75, 2368-2379.

Rolo, A.P., Oliveira, P.J., Moreno, A.J.M., Palmeira, C.M., 2000. Bile acids affect liver mitochondrial bioenergetics: possible relevance for cholestasis therapy. Toxicol. Sci. 57, 177-185.

Rolo, A.P., Oliveira, P.J., Moreno, A.J.M., Palmeira, C.M., 2001. Protective effect of carvedilol on chenodeoxycholate induction of the permeability transition pore. Biochem. Pharmacol. 61, 1449-1454.

Schaffner, F., Bacchin, P.G., Hutterer, F., et al., 1971. Mechanism of cholestasis. 4. Structural and biochemical changes in the liver and serum in rats after bile duct ligation. Gastroenterology 60, 888-897.

Scholmerich, J., Becher, M.S., Schmidt, K., et al., 1984. Influence of hydroxylation and conjugation of bile salts on their membrane-damaging properties - studies on isolated hepatocytes and lipid membrane vesicles. Hepatology 4, 661-666.

Schroder, O., Rathner, W., Caspary, W.F., Stein, J., 1996. Bile acidinduced increase of rat colonic apical membrane fluidity and proton permeability. Z. Gastroenterol. 34, 365-370.

Sokol, R.J., Devereaux, M., Khandwala, R., O'Brien, K., 1993. Evidence for involvement of oxygen free radicals in bile acid toxicity to isolated rat hepatocytes. Hepatology 17, 869-881.

Wallace, K.B., Starkov, A.A., 2000. Mitochondrial targets of drug toxicity. Annu. Rev. Pharmacol. Toxicol. 40, 353-388.

Yue, T.-L., McKenna, P.J., Lysko, P.J., et al., 1994. SB 211475, a metabolite of carvedilol, a novel antihypertensive agent, is a potent antioxidant. Eur. J. Pharmacol. 251, 237-243.

Zhao, D.L., Hirst, B.H., 1990. Bile salt-induced increases in duodenal brush-border membrane proton permeability, fluidity, and fragility. Dig. Dis. Sci. 35, 589-595.

Zoratti, M., Szabo, I., 1995. The mitochondrial permeability transition. Biochim. Biophys. Acta 1241, 139-176. 